Rev, Elev. Méd. vét. Pays trop., 1975, 28 (2) : 133-136

\title{
Essais d'induction d'une résistance acquise à l'infestation par Fasciola gigantica
}

\author{
par J.-M. BLANCOU (*), A. BOUCHET (**), P. DAYNES $\left(^{* * *}\right)$
}

\section{RESUME}

\begin{abstract}
Une primo-infestation de 22 zébus par Fasciola gigantica semble créer une résistance chez ces animaux par rapport aux témoins : 29 p. 100 de douves de moins entre les deux groupes.

Mais la seconde infestation n'est réduite que si elle a lieu au moins 180 jours après la première. Cette résistance semble avoir une base immunologique.
\end{abstract}

Les essais d'induction d'une résistance acquise à la fasciolose sont assez nombreux depuis quelques années, et concernent généralement des animaux de laboratoire (lapins, souris), des moutons ou des bovins infestés par Fasciola hepatica. Leurs résultats, en ce qui concerne les taurins, sont intéressants $(4,5,6,7)$. Nous avons donc exécuté des essais de même type, mais sur des zébus infestés par Fasciola gigantica.

C'est un fait d'observation courante que les bovins nés en zone d'enzootie parasitaire sont moins sensibles à la maladie que ceux introduits récemment dans ces zones.

A Madagascar, le phénomène fut observé avec encore plus de netteté puisque la fasciolose ne s'y est révélée qu'en 1966 et a atteint des zones traditionnellement indemnes. Les taux de mortalité observés lors des primoinfestations (1966) se sont révélés supérieurs à ceux notés les années suivantes dans les mêmes zones (2).

Région de Recherches Vétérinaires de Madagascar.

(*) Adresse actuelle : Laboratoire National de l'Elevage, B.P. n* 2057, Dakar-Hann, Sénégal.

(**) Laboratoire Pfizer, 37400 Amboise, France.

(***) Chemin du Maluan, 06800 Cagnes-sur-Mer, France.
Il paraissait donc possible de reproduire expérimentalement le phénomène naturel, et instructif de le quantifier.

\section{Animaux}

\section{MATERIEL}

Quarante quatre zébus malgaches, âgés de 3 à 6 ans ont été utilisés. Tous ces animaux provenaient d'une région totalement indemne de fasciolose et ont été soumis à une double coproscopie de contrôle avant infestation.

\section{Matériel d'infestation}

Il est constitué par des métacercaires de Fasciola gigantica. Ces métacercaires sont obtenues à partir de limnées naturellement infestées, récoltées dans la région, et mises en «émission" sous l'influence d'un stress lumineux et thermique. Les métacercaires sont récoltées sur des bandelettes plastiques permettant un dénombrement aisé; elles sont soumises ensuite à une période de "maturation " à la température du laboratoire (entre 22 et $28^{\circ} \mathrm{C}$ ), puis stockées au réfrigérateur. Au moment de l'emploi, les métacercaires sont dénombrées, les bandelettes sont incluses dans une boulette de 
pâte (farine + eau) puis administrées de force aux animaux.

\section{Principe}

\section{METHODES}

Infestation avec une forte quantité de métacercaires des bovins ayant reçu au préalable une infestation légère.

Cette seconde infestation a lieu, soit 90 jours, soit 180 jours après la primo-infestation.

Des animaux témoins n'ayant pas reçu de primo-infestation permettent de comparer les résultats : ceux-ci sont jugés d'après les lésions hépatiques et le nombre de douves retrouvées dans chaque foie.

En cours d'expérience, l'infestation est contrôlée par la détermination du taux des éosinophiles.

\section{Protocole de lexpérience}

\section{Infestation}

Quatre lots de onze animaux sont constitués. Ils sont infestés aux dates et avec les quantités de métacercaires indiquées dans le tableau I.

\section{Contrôle de l'évolution de l'infestation}

L'évolution est contrôlée par la détermination du taux des éosinophiles.

\section{Contrôle des résultats de l'infestation}

Lors de l'abattage des animaux, les foies sont examinés et toutes les douves qu'ils contiennent sont libérées dans un bain d'eau physiologique à $37^{\circ}$, puis dénombrées.

\section{RESULTATS}

1. Contrôle de l'évolution de l'infestation: Pourcentage d'éosinophiles.

Ils sont rapportés dans le tableau II.

2. Contrôle des résultats de l'ințestation: Dénombrement des douves selon les lots.

Ils figurent dans le tableau III.

\section{DISCUSSION - CONCLUSION}

L'étude statistique des résultats du tableau de dénombrement des douves, par analyse de variance à effets fixes et un facteur contrôlé, ne montre pas de différence entre les quatre lots au seuil $P=0,05$. En effet $F=2,12$ (alors que $F 0,05=2,86$ ) : ceci est probablement dû à la grande variabilité du nombre de douves par foie au sein d'un même lot.

Néanmoins, une différence existe au seuil $\mathbf{P}=0,1$ et entre le lot témoin 2000 et le lot réinfesté 180 jours après une primo-infestation légère : on dénombre 29 p. 100 de douves de moins dans le lot primo-infesté.

Par contre, lorsque la réinfestation est précoce $(\mathrm{J}+90)$ non seulement il n'existe aucune protection mais les animaux semblent avoir été en état de moindre résistance et s'être parasités plus sévèrement.

Nos conclusions rejoignent celles de HORCHNER (5) ROSS (6), DOYLE (3) sur les taurins réinfestés par Fasciola hepatica: ces auteurs signalent une diminution de 16 p. 100 à 56 p. 100 de la population de douves, à condition que la seconde infestation soit pratiquée au moins 17 semaines après la première.

Le mécanisme intime de la résistance induite par la primo-infestation est encore mal connu. J. EUZEBY (4) a examiné les différentes hypothèses actuelles à ce sujet, et attribue une importance relativement plus grande aux processus mécaniques (barrière des lésions de fibrose créées par la primo-infestation) qu'aux processus immunologiques. Mais l'un n'exclut pas l'autre et la genèse de la fibrose est peutêtre d'ordre immunologique.

Afin de confirmer, ou d'infirmer cette hypothèse nous avons effectué l'expérience complémentaire suivante :

Six bouvillons ont reçu, par voie sous-cutanée aux jours $\mathbf{J}_{0}$ et $\mathbf{J}_{45}$, un mélange ainsi composé :

- Broyat de Fasciola gigantica adulte, trypsiné * : $50 \mathrm{mg}$ (poids sec).

- Huile de paraffine et eau distillée stérile $(1 / 4): 1,5 \mathrm{ml}$.

$\mathrm{Au}$ jour $\mathrm{J}_{170}$, ils ont été infestés, ainsi que deux témoins neufs, par 1100 métacercaires. A l'autopsie $\left(J_{260}\right)$ le nombre moyen de douves

(*) Broyat total de douves adultes lavées, digéré 2 heures à 370 dans un égal volume de trypsine à 1 p. 100 : cette méthode est employée dans la préparation de certains antigènes bactériens. 
TABLEAU N $N^{\circ}$ I

\begin{tabular}{|c|c|c|c|c|c|}
\hline \multirow{2}{*}{$L$ o $t$} & \multicolumn{2}{|c|}{ Première infestation } & \multicolumn{2}{|c|}{ Seconde infestation } & \multirow{2}{*}{$\begin{array}{c}\text { Date de } \\
\text { l'autopsie }\end{array}$} \\
\hline & $\begin{array}{l}\text { Quantitē de } \\
\text { Métacercalres }\end{array}$ & Date & $\begin{array}{l}\text { Quantité de } \\
\text { Métacercaires }\end{array}$ & Date & \\
\hline Témoin "200" & 200 & $\mathrm{~J}$ & - & - & $J+200$ \\
\hline Témoin "2 000" & 2000 & $\mathrm{~J}$ & - & - & $J+130$ \\
\hline Rêinfestês "J + 90" & 200 & $\mathrm{~J}$ & 2000 & $\mathrm{~J}+90$ & $J+200$ \\
\hline Rêinfestús "J + 180" & 200 & $\mathrm{~J}$ & 2000 & $\mathrm{~J}+180$ & $\begin{array}{c}\mathrm{J}+310 \\
(180+130)\end{array}$ \\
\hline
\end{tabular}

$\mathrm{J}=$ jour de J'infestation.

TABLEAU $N^{0} I I$

\begin{tabular}{|l|c|c|c|c|c|}
\hline & $\begin{array}{c}\text { Avant } \\
\text { infestation }\end{array}$ & $\mathrm{J}+30$ & $\mathrm{~J}+60$ & \multicolumn{2}{|c|}{ Après réinfestation } \\
\cline { 2 - 5 } $\begin{array}{l}\text { Témoin "200" } \\
\text { Métacercaires }\end{array}$ & 5,39 & 9,18 & $\mathrm{~J}+30$ & $\mathrm{~J}^{\prime}+60$ \\
\hline $\begin{array}{l}\text { Témoin "2 000" } \\
\text { Métacercaires }\end{array}$ & 5,85 & 12,01 & 12,29 & & \\
\hline $\begin{array}{l}\text { Réinfestês à } \\
\mathrm{J}+90\end{array}$ & 3,81 & 11,21 & 10,33 & 13,04 & 12,21 \\
\hline $\begin{array}{l}\text { Réinfestês à } \\
\mathrm{J}+180\end{array}$ & 7,45 & 11,27 & 9,87 & 13,77 & 11,31 \\
\hline
\end{tabular}

$J=$ jour de $\mathrm{I}^{\prime}$ infestation $; \mathrm{J}^{\prime}$ = jour de la réinfestation.

TABLEAU $N^{\circ}$ III

\begin{tabular}{|c|c|c|c|c|}
\hline \multirow{2}{*}{$\begin{array}{c}\text { Numéro } \\
\text { des animaux }\end{array}$} & \multicolumn{2}{|c|}{ Nombre de douves par animal } \\
\cline { 2 - 5 } & Témoin "200" & Témoin "2 000" & Réinfestếs "J + 90" & Réinfestés "J + 180" \\
\hline 2 & 45 & 44 & 12 & 0 \\
\hline 3 & 13 & 19 & 125 & 13 \\
\hline 4 & 49 & 0 & 19 & 2 \\
\hline 5 & 25 & 18 & 20 & 51 \\
\hline 6 & 5 & 38 & 151 & 61 \\
\hline 7 & 23 & 69 & 23 & 16 \\
\hline 8 & 16 & 45 & 29 & 13 \\
\hline 9 & 6 & 8 & 95 & 21 \\
\hline 10 & 8 & 34 & 50 & 67 \\
\hline 11 & 19 & 11 & 5 & 28 \\
\hline Total & 21 & 100 & 35 & 272 \\
\hline Moyenne & 230 & 386 & 594,00 & 24,72 \\
\hline
\end{tabular}


chez ces deux témoins était de 199, contre 131 chez les sujets ayant reçu l'antigène distomien, et les lésions de cirrhose étaient nettement moins accusées chez ces derniers.
Cette expérience, quoique sans valeur statistique, semblerait confirmer le rôle des phénomènes immunologiques dans la résistance acquise à la réinfestation.

\section{SUMMARY}

Trials to induce an acquired immunity against the infestation by Fasciola gigantica

Twenty two zebu cattle previously infected by metacercariae of Fasciola gigantica showed fewer successful infection (29 p. 100 lesser) than did controls receiving the initial infection only.

The reinfection is reduced only if animals are challenged 180 days after the initial infection: this resistance seems to be an immunity phenomenon.

\section{RESUMEN}

Ensayos de inducción de una resistencia adquirida a la infestación por Fasciola gigantica

Una primoinfestación de 22 cebues por Fasciola gigantica parece provocar una resistencia en estos animales respecto a testigos : 23 p. 100 de distomas menos que entre los dos grupos.

Pero se reduce la segunda infestación sólo si ocurre por lo menos 180 días despues de la primera. Dicha resistencia parece tener una base inmunologica.

\section{BIBLIOGRAPHIE}

1. BLANCOU (J.), BOUCHET (A.), DAYNES (P.). Etude sur l'allergie, les anticorps précipitants et les anticorps fixant le complément au cours de l'infestation des bovins par Fasciola gigantica Rev. Elev. Méd. vét. Pays trop. 1971, 24 (3) : 373-379.

2. DAYNES (P.), BOUCHET (A.). La distomatose des ruminants à Fasciola gigantica. Terre malgache, 1972, 14: 123-150.

3. DOYLE (J.J.). Acquired immunity to experimental infection with Fasciola hepatica in cattle. Res. vet. Sci., 1971, 12 (6): 527-534.

4. EUZEBY (J.). Les maladies vermineuses des animaux domestiques et leurs incidences sur la pathologie humaine. Tome II. Livre 1. Paris, Vigot frères, 1971.

5. HORCHNER (F.). Clinical and immunological studies on experimental Fasciola hepatica infection in cattle. Berl. Münch. tierärztl Wschr., 1969, 82 : 204-208.

6. ROSS (J. G.). Experimental infection of cattle with Fasciola hepatica. High level single infections in calves. The production of an acquired self cure by challenge infection. J. Helminth., 1967, 41: 217228.

7. ROSS (J. G.). Studies of immunity to Fasciola hepatica: acquired immunity in cattle, sheep and rabbits following natural infection and vaccine procedure. J. Helminth., 1967, 41 : 393-399. 